\title{
ON THE RELATIVE SOLVABILITY OF CERTAIN INVERSE MONOIDS
}

\author{
EDITE CORDEIRO AND MANUEL DELGADO
}

\begin{abstract}
The notion of kernel of a finite monoid relative to a pseudovariety of groups can be used to define relative solvability of monoids in a similar way to that the notion of derived subgroup can be used to define solvable group. In this paper we study the solvability of certain inverse monoids relative to pseudovarieties of abelian groups.
\end{abstract}

Keywords: Inverse monoid; pseudovariety; (relative) abelian kernel; (relative) solvability Communicated by Mikhail Volkov.

\section{InTRODUCTION}

A pseudovariety of groups is a class of finite groups closed under the formation of subgroups, homomorphic images and finite direct products. The notion of $\mathrm{H}$-solvable monoid, with $\mathrm{H}$ a pseudovariety of groups, was introduced by Fernandes and the second author in [6]: a finite monoid is $\mathrm{H}$-solvable if iterating the computation of the relative $\mathrm{H}$-kernel one eventually arrives to the submonoid generated by the idempotents. As the $A b$-kernel of a finite group is its derived subgroup, we get that a finite group is Ab-solvable if and only if it is solvable in the usual sense. Generalizations of the main result given in [6], so as related consequences, have been obtained in two subsequent papers $[8,7]$. The most general result concerning $\mathrm{H}$-solvability, obtained in [8], states that "a semigroup $S$ is $\mathrm{H}$-solvable if and only if, for each idempotent $e \in S$, there is a subnormal series with smallest element the maximal subgroup at $e$ of the idempotent-generated subsemigroup of $S$ and largest element the maximal subgroup of $S$ at $e$ such that the successive quotients belong to H". At the pseudovariety level these notions (iterating the computation of relative kernels or Mal'cev products) led to interesting consequences. For example, we have that $E A=A^{\omega}(m)$.

There exist monoids generated by idempotents and containing non-solvable subgroups. Therefore, the notion of "Ab-solvable monoid" does not coincide with the notion of "monoid whose subgroups are solvable" which is sometimes referred in the literature as "solvable monoid" [15]. That for inverse semigroups both notions coincide follows from the result recalled above (in fact, it already follows from the (less general) result stated in [6]).

The pseudovarieties of groups that we will be dealing with in this paper are decidable pseudovarieties of abelian groups. These are in bijection with recursive supernatural numbers. An algorithm to compute kernels of finite monoids relative to any of these pseudovarieties was given by Steinberg [14]. (See also [3].) Concrete descriptions of relative abelian kernels of some inverse transformation monoids have been given in a joint work with Fernandes [1]. In some sense, that paper is continued here, since we consider iterations of the relative abelian kernel operator. The key ingredient is stated in Section 3 and says that any relative abelian kernel of a finite group is generated by its derived subgroup and some appropriate powers. In [1] such a statement had been obtained for pseudovarieties of abelian groups corresponding to finite supernatural numbers. 
Section 2 contains notation and preliminaries to be used throughout the paper. Notation, so as results, concerning some inverse transformation monoids is left to the last section, since it is just used there.

The main result of this paper is proved in Section 3. It states that the kernel of a finite group relative to a pseudovariety corresponding to a recursive supernatural number $\pi$ is generated by its derived subgroup and powers whose exponents are the greatest common divisors of $\pi$ and the order of the group.

In Section 4 we give some applications, by computing (iterations of) relative abelian kernels of some groups.

Section 5 is dedicated to the solvability of some inverse monoids, so as to the length of the chains of relative abelian kernels.

\section{NotATION AND PRELIMINARY RESUlts}

The notion of supernatural number (which may be seen as an extension of the notion of natural number) is well known and used in various areas (see, for instance, [13] and [14]). Due to its importance in this paper, we recall it in some detail in the first subsection. In the other subsections are recalled definitions and some facts concerning abelian groups, pseudovarieties of abelian groups an relative kernels.

2.1. Supernatural numbers. A supernatural number is a formal product of the form

$$
\prod_{p} p^{n_{p}}
$$

where $p$ ranges over all prime positive integers and $n_{p}$ is either a natural number or $+\infty$. By extending the addition of natural numbers, we assign to $\mathbb{N} \cup\{+\infty\}$ the structure of a monoid with zero $(+\infty$ is the zero). By defining

$$
\prod_{p} p^{\alpha_{p}} \prod_{p} p^{\beta_{p}}=\prod_{p} p^{\alpha_{p}+\beta_{p}}
$$

we get assigned to the set of supernatural numbers a structure of commutative monoid which, in view of the fundamental theorem of arithmetic, extends the corresponding structure for positive integers.

We consider that $+\infty$ is greater than any integer. Given a supernatural number $\pi$, we denote by $e_{p}(\pi)$ the exponent $n_{p}$ of the prime $p$ occurring in $\pi$. Next we define some notions that are obvious generalizations of the corresponding notions for positive integers.

We say that a supernatural number $\pi$ divides a supernatural number $\rho$, and write $\pi \mid \rho$, if $e_{p}(\pi) \leq e_{p}(\rho)$ for all prime $p$. Note that $\pi \mid \rho$ if and only if there exists a supernatural number $\gamma$ such that $\rho=\pi \gamma$. We can use the notion of division to define greatest common divisor and least common multiple of two supernatural numbers. Alternatively, in analogy with what is commonly done when dealing with natural numbers, we can define these notions using the following formulas:

$$
\left\{\begin{array}{l}
\operatorname{gcd}(\pi, \rho)=\prod_{p} p^{\min \left\{e_{p}(\pi), e_{p}(\rho)\right\}} \\
\operatorname{lcm}(\pi, \rho)=\prod_{p} p^{\max \left\{e_{p}(\pi), e_{p}(\rho)\right\}} .
\end{array}\right.
$$

The set

$$
\operatorname{supp}(\pi)=\left\{p \mid e_{p}(\pi)>0\right\}
$$

is said to be the support of $\pi$. A supernatural number of finite support such that all the exponents of the primes occurring in it are finite will be called indistinctively a "positive 
integer", "natural number" or "finite supernatural number". Note that in this context a natural number can not be zero.

Given a supernatural number $\pi$, we denote by $\operatorname{div}(\pi)$ the set of its finite divisors and say that $\pi$ is recursive if $\operatorname{div}(\pi)$ is recursive. For example, supernatural numbers of finite support are recursive.

The following remark follows from the definitions.

Remark 2.1. If $a, b \in \operatorname{div}(\pi)$, then $\operatorname{lcm}(a, b) \in \operatorname{div}(\pi)$.

Throughout this paper, $\pi$ will always denote a (possibly infinite) recursive supernatural number.

2.2. Finite abelian groups. The notation $\mathrm{C}_{n}$ will be used for the cyclic group $\mathbb{Z} / n \mathbb{Z}$ of order $n$. The following well known observation will be of use in this paper.

Remark 2.2. Let $n$ and $r$ be positive integers. If $g$ is a generator of the cyclic group $\mathrm{C}_{n}$, then $\left\langle g^{r}\right\rangle$, the cyclic subgroup generated by $g^{r}$, has order $n / \operatorname{gcd}(r, n)$.

The finite cyclic groups are the building blocks of the finite abelian groups as says the next result, which is known as the Fundamental Theorem of Finite Abelian Groups. A proof can be found in any basic algebra textbook (see, for instance, [11, 9]).

Theorem 2.3. Let $G$ be a finite abelian group. Then $G$ is isomorphic to a finite direct product of finite cyclic groups

$$
\mathrm{C}_{m_{1}} \times \mathrm{C}_{m_{2}} \times \cdots \times \mathrm{C}_{m_{k}}
$$

where $m_{i} \mid m_{i-1}$, with $2 \leq i \leq n$.

We will refer the form (2) as the canonical form of the abelian group $G$ given by the fundamental theorem of finite abelian groups. The $m_{i}$ 's are known as the torsion coefficients of $G$.

Using the fact that the direct product of two cyclic groups of coprime orders is a cyclic group, we can combine cyclic factors of coprime orders and write the group in other forms. One of them is

$$
\mathrm{C}_{p_{1}^{n_{1}}} \times \mathrm{C}_{p_{2}^{n_{2}}} \times \cdots \times \mathrm{C}_{p_{k}^{n_{k}}}
$$

where the $p_{i}$ 's are primes, not necessarily distinct Note that the powers $p_{1}^{n_{1}}, p_{2}^{n_{2}}, \ldots, p_{k}^{n_{k}}$ are unique.

2.3. Pseudovarieties of abelian groups. To a supernatural number $\pi$ we associate the pseudovariety of abelian groups $\mathrm{H}_{\pi}$ of all finite abelian groups whose torsion coefficients divide $\pi$ (i.e. $\mathrm{H}_{\pi}=\left\langle\left\{\mathrm{C}_{m} \mid m \in \operatorname{div}(\pi)\right\}\right\rangle$ ). For example, to the supernatural number $\prod p^{+\infty}$, where $p$ runs over all prime positive integers, is associated the pseudovariety $\mathrm{Ab}$ of all finite abelian groups. Notice that the pseudovariety of abelian groups associated to a natural number $k$ is just $\left\langle\mathrm{C}_{k}\right\rangle$, the pseudovariety generated by the cyclic group of order $k$. It follows that $\mathrm{H}_{\pi}$ is the least pseudovariety containing the pseudovarieties $\mathrm{H}_{k}$, where $k$ runs over the natural divisors of $\pi$, that is, $\mathrm{H}_{\pi}=\bigvee_{k \in \operatorname{div}(\pi)} \mathrm{H}_{k}$.

The pseudovarieties of abelian groups associated to recursive supernatural numbers are precisely those that are decidable. We refer the reader to Steinberg's paper [14] for details. 
2.4. Relative kernels. A relational morphism of monoids $\tau: M \rightarrow N$ is a function from $M$ into the power set of $N$, such that $\tau\left(s_{1}\right) \neq \emptyset$ and $\tau\left(s_{1}\right) \tau\left(s_{2}\right) \subseteq \tau\left(s_{1} s_{2}\right)$, for all $s_{1}, s_{2} \in M$. Moreover, $1 \in \tau(1)$.

Let $\mathrm{H}$ be a pseudovariety of groups. The $\mathrm{H}$-kernel of a finite monoid $M$ is the set

$$
\mathrm{K}_{\mathrm{H}}(M)=\bigcap_{\tau: M \rightarrow G ; G \in \mathrm{H}} \tau^{-1}(1)
$$

(the intersection is taken over all relational morphisms $\tau: M \rightarrow G$, with $G \in \mathrm{H}$ ). It is easily seen to be a submonoid of $M$ containing the idempotents.

As an example, we have that, for a finite group $G, \mathrm{~K}_{\mathrm{Ab}}(G)=G^{\prime}$, the derived subgroup of $G$. This is a consequence of the following result.

Proposition 2.4. [4, Proposition 9.6] Let $G$ be a finite group and let $\mathrm{H}$ be a pseudovariety of groups. The $\mathrm{H}$-kernel of $G$ is the smallest normal subgroup of $G$, such that $G / \mathrm{K}_{\mathrm{H}}(G) \in$ $\mathrm{H}$.

Let $M$ be a finite monoid. As the $\mathrm{H}$-kernel of $M$ is a submonoid, one can iterate the process. This idea leads to the following recursive definition of $\mathrm{K}_{\mathrm{H}}^{n}$ :

- $\mathrm{K}_{\mathrm{H}}^{0}(M)=M$;

- $\mathrm{K}_{\mathrm{H}}^{n}(M)=\mathrm{K}_{\mathrm{H}}\left(\mathrm{K}_{\mathrm{H}}^{n-1}(M)\right)$, for $n \geq 1$.

We adopt the terminology introduced by Fernandes and the second author [6] and say that a finite monoid $M$ is $\mathrm{H}$-solvable if, for some non-negative integer $n, \mathrm{~K}_{\mathrm{H}}^{n}(M)=\langle E(M)\rangle$ where $\langle E(M)\rangle$ is the submonoid generated by the set $E(M)$ of the idempotents of $M$.

Given a finite $\mathrm{H}$-solvable monoid $M$, we define the $\mathrm{H}$-kernel chain length of $M$ as the least natural number $n$ such that $\mathrm{K}_{\mathrm{H}}^{n}(M)=\langle E(M)\rangle$ and denote it by $\ell_{\mathrm{H}}(M)$. Note that a finite group is Ab-solvable precisely when it is solvable in the usual sense, as follows from the fact that the abelian kernel of a finite group is its derived subgroup. Note also that, for a group $G, \ell_{\mathrm{Ab}}(G)$ is nothing more than the derived length of $G$.

\section{RELATIVE ABELIAN KERNELS OF FINITE GROUPS}

Given a finite group $G$ and a natural number $k$, denote by $G^{[k]}$ the subgroup of $G$ generated by the commutators of $G$ (that is, the elements of the form $x y x^{-1} y^{-1}, x, y \in G$ ) and by the $k$-powers of $G$ (that is, the elements of the form $x^{k}, x \in G$ ). In other words, $G^{[k]}$ is the smallest subgroup of $G$ containing the derived subgroup $G^{\prime}$ and the $k$-powers. Clearly, $G^{[1]}=G$.

It is convenient to observe the following:

Lemma 3.1. Let $G$ be a finite group of order $n$ and let $k$ be a positive integer. Then

$$
G^{[k]}=G^{[\operatorname{gcd}(k, n)]} \text {. }
$$

Proof. As $\operatorname{gcd}(k, n)$ divides $k$, it follows immediately that $G^{[k]} \subseteq G^{[\operatorname{gcd}(k, n)]}$.

To prove the converse inclusion we use the fact that there exist integers $r$ and $s$ such that $\operatorname{gcd}(k, n)=r k+s n$. Let $g^{\operatorname{gcd}(k, n)} \in G^{[\operatorname{gcd}(k, n)]}$. Then $g^{\operatorname{gcd}(k, n)}=g^{r k+s n}=g^{r k} \in G^{[k]}$. It follows that there is a set of generators of $G^{[\operatorname{gcd}(k, n)]}$ contained in $G^{[k]}$, and therefore $G^{[\operatorname{gcd}(k, n)]} \subseteq G^{[k]}$.

The main result of this section (Theorem 3.11) extends to the supernatural setting a result of Fernandes and the authors ([1, Proposition 1.17]) which, taking into account the previous lemma, may be stated as follows: 
Proposition 3.2. For a finite group $G$ and a natural number $k$, we have:

$$
\mathrm{K}_{\mathrm{H}_{k}}(G)=G^{[\operatorname{gcd}(k,|G|)]} .
$$

Next we will work towards the proof of Theorem 3.11.

Lemma 3.3. Let $a$ and $b$ be positive integers and let $m=\operatorname{lcm}(a, b)$. The pseudovariety generated by the cyclic groups $\mathrm{C}_{a}$ and $\mathrm{C}_{b}$ is precisely the pseudovariety $\mathrm{H}_{m}$ of abelian groups generated by the cyclic group $\mathrm{C}_{m}$, that is $\mathrm{H}_{a} \vee \mathrm{H}_{b}=\mathrm{H}_{m}$.

Proof. Let us consider factorizations $a=p_{1}^{\alpha_{1}} p_{2}^{\alpha_{2}} \ldots p_{k}^{\alpha_{k}}$ and $b=p_{1}^{\beta_{1}} p_{2}^{\beta_{2}} \ldots p_{k}^{\beta_{k}}$ of $a$ and $b$, where the $p_{i}$ are distinct primes, for $i \in\{1, \ldots, k\}$. It follows from the fact that the direct product of two cyclic groups of coprime orders is cyclic that, for $a$ and $b$ as above, we have: $\mathrm{C}_{a} \simeq \mathrm{C}_{p_{1}^{\alpha_{1}}} \times \mathrm{C}_{p_{2}^{\alpha_{2}}} \times \cdots \times \mathrm{C}_{p_{k}^{\alpha_{k}}}, \quad \mathrm{C}_{b} \simeq \mathrm{C}_{p_{1}^{\beta_{1}}} \times \mathrm{C}_{p_{2}^{\beta_{2}}} \times \cdots \times \mathrm{C}_{p_{k}^{\beta_{k}}}$ and

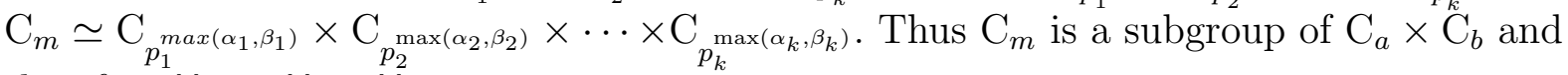
therefore $\mathrm{H}_{m} \subseteq \mathrm{H}_{a} \vee \mathrm{H}_{b}$.

In order to prove the reverse inclusion, note that as $a \mid m$ and $b \mid m$, we have that $\mathrm{C}_{a}$ and $\mathrm{C}_{b}$ are subgroups of $\mathrm{C}_{m}$. It follows that $\left\{\mathrm{C}_{a}, \mathrm{C}_{b}\right\} \subseteq \mathrm{H}_{m}$ and therefore $\mathrm{H}_{a} \vee \mathrm{H}_{b} \subseteq \mathrm{H}_{m}$.

By induction, one immediately gets the following:

Corollary 3.4. Let $a_{1}, \ldots, a_{n}$ be positive integers. Then

$$
\bigvee_{1 \leq i \leq n} \mathrm{H}_{a_{i}}=\mathrm{H}_{\operatorname{lcm}\left(a_{1}, \ldots, a_{n}\right)} \text {. }
$$

Proposition 3.5. The set $\mathrm{V}=\bigcup_{k \in \operatorname{div}(\pi)} \mathrm{H}_{k}$ is a pseudovariety containing $\mathrm{H}_{\pi}$.

Proof. The set $\mathrm{V}$ is closed under taking subgroups and homomorphic images, since for any group $G \in \mathrm{V}$ there exists $k$ such that $G \in \mathrm{H}_{k}$. Note that, as $\mathrm{H}_{k}$ is a pseudovariety, subgroups and homomorphic images of $G$ belong to $\mathrm{H}_{k}$. In order to show that $\mathrm{V}$ is in fact a pseudovariety, it remains to prove that $\mathrm{V}$ is closed under finite direct products.

Let $G_{1}, G_{2}, \ldots, G_{n} \in \mathrm{V}$. There exist $k_{1}, k_{2}, \ldots, k_{n} \in \operatorname{div}(\pi)$ such that $G_{i} \in \mathrm{H}_{k_{i}}$, for $1 \leq i \leq n$. By Corollary 3.4, $G_{1} \times \cdots \times G_{n} \in \mathrm{H}_{\mathrm{lcm}\left(k_{1}, \ldots, k_{n}\right)}$. From Remark 2.1, one easily concludes that $G_{1} \times \cdots \times G_{n} \in \mathrm{V}$, and therefore $\mathrm{V}$ is closed under finite direct products.

That $V$ contains $\mathrm{H}_{\pi}$ follows from the fact that it contains a set $\left\langle\left\{\mathrm{C}_{k} \mid k \in \operatorname{div}(\pi)\right\}\right\rangle$ of generators of $\mathrm{H}_{\pi}$.

Since $\mathrm{H}_{k} \subseteq \mathrm{H}_{\pi}$, for any $k \in \operatorname{div}(\pi)$, it follows from previous proposition that $\mathrm{H}_{\pi}=$ $\bigcup_{k \in \operatorname{div}(\pi)} \mathrm{H}_{k}$.

In order to state a sharper result, we introduce the notation $\Delta_{\pi, n}$ to denote the set of integers that are simultaneously divisors of the supernatural number $\pi$ and multiples of the natural number $n$.

Example 3.6. Let $\pi=2^{+\infty} \cdot 3^{7} \cdot 7^{+\infty}$ and $n=2^{3} \cdot 3^{4}$. Then

$$
\Delta_{\pi, n}=\left\{2^{i+3} \cdot 3^{j} \cdot 7^{k} \mid 4 \leq j \leq 7 ; i, k \geq 0\right\} .
$$

Corollary 3.7. Let $n \in \operatorname{div}(\pi)$. Then $\mathrm{H}_{\pi}=\bigcup_{k \in \Delta_{\pi, n}} \mathrm{H}_{k}$.

Proof. Since $\mathrm{H}_{\pi}=\bigcup_{k \in \operatorname{div}(\pi)} \mathrm{H}_{k}$, we just have to show that $\bigcup_{k \in \operatorname{div}(\pi)} \mathrm{H}_{k}=\bigcup_{k \in \Delta_{\pi, n}} \mathrm{H}_{k}$. Clearly the right hand side is contained in the left hand side. The reverse inclusion follows from the fact that, for $k \in \operatorname{div}(\pi), \mathrm{H}_{k} \subseteq \mathrm{H}_{\mathrm{lcm}(k, n)}$. Note that, using Remark 2.1, $\operatorname{lcm}(k, n) \in \Delta_{\pi, n}$.

For the next results of this section, we fix a supernatural number $\pi$, a finite group $G$ and use the notation $d=\operatorname{gcd}(\pi,|G|)$. 
Lemma 3.8. If $k \in \Delta_{\pi, d}$, then $\operatorname{gcd}(k,|G|)=d$.

Proof. Note that if $k \in \operatorname{div}(\pi)$, then $\operatorname{gcd}(k,|G|) \mid d$. Noting that $|G|$ is a multiple of $d$, we have that if $k$ is a multiple of $d$, then $d \mid \operatorname{gcd}(k,|G|)$. We have observed that if $k \in \Delta_{\pi, d}$, then $d \mid \operatorname{gcd}(k,|G|)$ and $\operatorname{gcd}(k,|G|) \mid d$. The result follows.

Corollary 3.9. If $k \in \Delta_{\pi, d}$, then $G^{[k]}=G^{[d]}$.

Proof. By Lemma 3.1, we have that $G^{[k]}=G^{[\operatorname{gcd}(k,|G|)]}$. The result follows from previous lemma.

Lemma 3.10. Let $\mathrm{V}_{i}(i \geq 1)$ be pseudovarieties of groups such that $\mathrm{V}=\bigcup_{i \geq 1} \mathrm{~V}_{i}$ is a pseudovariety. Then

$$
\bigcap_{i \geq 1} \mathrm{~K}_{\mathrm{V}_{i}}(G)=\mathrm{K}_{\mathrm{V}}(G)
$$

Proof. As $\mathrm{V}$ contains each $\mathrm{V}_{i}(i \geq 1)$, we have that $\mathrm{K}_{\mathrm{V}}(G)$ is contained in each $\mathrm{K}_{\mathrm{V}_{i}}(G)$ and, therefore, also in the intersection.

To prove the converse inclusion, observe that $g \in \bigcap_{i>1} \mathrm{~K}_{\mathrm{V}_{i}}(G)$ just means that $g \in$ $\tau^{-1}(1)$, for every relational morphism $\tau: G \leftrightarrow H$ where $H$ is a group in one of the $\mathrm{V}_{i}$. But this is nothing more than $g \in \mathrm{K}_{\mathrm{V}}(G)$.

As $\Delta_{\pi, d}$ is numerable, we can apply the previous lemma to $\mathrm{H}_{\pi}=\bigcup_{k \in \Delta_{\pi, d}} \mathrm{H}_{k}$ (Corollary 3.7), obtaining:

$$
\bigcap_{k \in \Delta_{\pi, d}} \mathrm{~K}_{\mathrm{H}_{k}}(G)=\mathrm{K}_{\mathrm{H}_{\pi}}(G) .
$$

By Proposition 3.2 and Corollary 3.9, we have, for $k \in \Delta_{\pi, d}$ :

$$
\mathrm{K}_{\mathrm{H}_{k}}(G)=G^{[k]}=G^{[d]} \text {. }
$$

Thus, Equation (4) reduces to

$$
G^{[d]}=\bigcap_{k \in \Delta_{\pi, d}}\left(G^{[d]}\right)=\mathrm{K}_{\mathrm{H}_{\pi}}(G) .
$$

We have proved the main result of this section.

Theorem 3.11. Let $G$ be a finite group and let $\pi$ be a supernatural number. Then

$$
\mathrm{K}_{\mathrm{H}_{\pi}}(G)=G^{[\operatorname{gcd}(\pi,|G|)]} .
$$

Remark 3.12. Note that $\operatorname{gcd}(\pi,|G|)$ only depends on $|G|$ and the supernatural number obtained from $\pi$ by considering the primes that divide $|G|$. Therefore, we can restrict the study of $\mathrm{H}_{\pi}$-kernels of finite groups to kernels of finite groups relative to pseudovarieties corresponding to supernatural numbers of finite support.

Suppose that $G$ is a finite group whose order is divisible by a prime $p$. It is well known (see Cauchy's theorem in any basic Algebra textbook) that $G$ has an element $x$ of order $p$. Suppose, in addition that $p$ does not divide $\pi$. This implies that $p$ does not divide $\operatorname{gcd}(|G|, \pi)$, and therefore that $x^{\operatorname{gcd}(|G|, \pi)}$ has order $p$. It follows that $x$ and $x^{\operatorname{gcd}(|G|, \pi)}$ generate the same cyclic group, that is, $\langle x\rangle=\left\langle x^{\operatorname{gcd}(|G|, \pi)}\right\rangle$. We have proved the following:

Proposition 3.13. Let $G$ be a finite group and suppose that there is a prime $p$ that divides $|G|$ but does not divide $\pi$. Then there exists an element $x \in G$ of order $p$ such that $\langle x\rangle \subseteq \mathrm{K}_{\mathrm{H}_{\pi}}(G)$. 
If $G$ satisfies the conditions of previous proposition, then so does $\mathrm{K}_{\mathrm{H}_{\pi}}(G)$. We can therefore conclude that $\mathrm{K}_{\mathrm{H}_{\pi}}^{i}(G)$ contains $\langle x\rangle$ and has order divisible by $p$, for all $i \geq 1$.

As a consequence we have the following:

Corollary 3.14. If $G$ is a group of even order and $\pi$ is odd, then $G$ is not $\mathrm{H}_{\pi}$-solvable.

Another immediate consequence is:

Corollary 3.15. If $G$ is a $\mathrm{H}_{\pi}$-solvable finite group, then all prime divisors of $|G|$ divide $\pi$.

The converse is not true in general. In fact, its truth would imply the solvability (i.e. the Ab-solvability) of all finite groups. (Recall that $\mathrm{Ab}=\mathrm{H}_{\prod p^{+\infty}}$, where $p$ runs over all prime positive integers.)

\section{Applications to ABelian, Dihedral AND Symmetric Groups}

Aiming to describe relative abelian kernels of certain finite monoids whose maximal subgroups are cyclic, dihedral and symmetric groups, Fernandes and the authors have computed in [1] relative abelian kernels of this kind of groups. We will give applications of Theorem 3.11 by giving new proofs of these results, as well as computing relative abelian kernels of abelian groups. Then we consider iterations of these computations. As we shall observe, most of them rely on the iterations of the relative abelian kernels of cyclic groups.

Recall that $G^{[k]}$ is the smallest subgroup of $G$ containing the derived subgroup $G^{\prime}$ and the $k$-powers. If $G$ is abelian, then $G^{[k]}$ is generated by the $k$-powers, since the only commutator of an abelian group is the neutral element. As in a abelian group the product of $k$-powers is a $k$-power, it is clear that if $A$ is a set of generators of an abelian group $G$, then $G^{[k]}=\left\langle\left\{g^{k} \mid g \in A\right\}\right\rangle$.

Throughout this section, $n>1$ is a fixed natural number.

\subsection{Relative abelian kernels.}

4.1.1. The case of a finite cyclic group. Let $\mathrm{C}_{n}=\langle g\rangle$ be the cyclic group of order $n$, generated by $g$, and let $d=\operatorname{gcd}(\pi, n)$. As $\mathrm{C}_{n}$ is abelian, we have that $\mathrm{C}_{n}^{[d]}=\left\langle g^{d}\right\rangle$. It is a cyclic group of order $n / d$, by Remark 2.2 .

4.1.2. The case of a finite dihedral group. Let $\mathrm{D}_{2 n}=\left\langle h, g \mid h^{2}=g^{n}=(g h)^{2}=1\right\rangle$ be the dihedral group of order $2 n$ and let $d=\operatorname{gcd}(\pi, 2 n)$. Note that the parity of $d$ coincides with the parity of $\pi$.

As $\left\langle g^{2}\right\rangle=\mathrm{D}_{2 n}^{\prime}$ (see, for instance, [9]), we have that $\left\langle g^{2}\right\rangle \subseteq \mathrm{D}_{2 n}^{[d]}$. It is easy to observe that $\mathrm{D}_{2 n}$ satisfies the relation $h g^{n-1}=g h$, thus the elements of $\mathrm{D}_{2 n}$ may be written in the form $g^{i}$ or $h g^{i}$, with $i \in\{1, \ldots, n\}$.

By induction on $i$, one can verify that, for $i \in\{1, \ldots, n\}$, the equality $\left(h g^{i}\right)^{2}=1$ holds. In fact, for $i=1$, we have $h g h g=h^{2} g^{n-1} g=h^{2} g^{n}=1$. Supposing now that the equality holds for $i-1$, we have $\left(h g^{i}\right)^{2}=h g^{i} h g^{i}=h g^{i-1} g h g^{i}=h g^{i-1} h g^{n-1} g^{i}=h g^{i-1} h g^{i-1}=1$, as we wanted to prove. As a consequence, we have that, if $d$ is even, then $\left(h g^{i}\right)^{d}=1$.

Next we will consider two subcases, according to the parity of $d$.

Subcase 1. Suppose that $d$ is even. Then $\left(h g^{i}\right)^{d}=1$ and therefore $\mathrm{D}_{2 n}^{[d]}=\mathrm{D}_{2 n}^{\prime}=\left\langle g^{2}\right\rangle$.

Subcase 2. If $d$ is odd, then there exists $r$ such that $d=2 r+1$. Thus $\left(h g^{i}\right)^{d}=$ $\left(h g^{i}\right)^{2 r+1}=h g^{i}$, for $i \in\{1, \ldots, n\}$. In particular, taking $i=n$ or $i=1$, we get that $h, h g \in G^{[d]}$. This implies that $g=h \cdot h g \in \mathrm{D}_{2 n}^{[d]}$ and therefore $\mathrm{D}_{2 n}^{[d]}=\mathrm{D}_{2 n}$. 
4.1.3. The case of a finite symmetric group. Let us consider the group $\mathcal{S}_{n}$ and let $d=$ $\operatorname{gcd}(\pi, n !)$. Observe that we are supposing that $n>1$, hence $n$ ! is even. Therefore, as in the case of the dihedral groups, we have that the parity of $d$ coincides with the parity of $\pi$. Recall that $\mathcal{S}_{n}^{\prime}=\mathcal{A}_{n}$.

Subcase 1. If $d$ is even and $\sigma \in \mathcal{S}_{n}$, then $\sigma^{d} \in \mathcal{A}_{n}$. As $\mathcal{S}_{n}^{\prime}=\mathcal{A}_{n}$, we get that $\mathcal{S}_{n}^{[d]}=\mathcal{A}_{n}=\mathcal{S}_{n}^{\prime}$

Subcase 2. If $d$ is odd and $\sigma \in \mathcal{S}_{n}$, then $\sigma^{d}$ has the parity of $\sigma$. Thus, as we are assuming $n>1, \mathcal{S}_{n}{ }^{[d]}$ can not be contained in $\mathcal{A}_{n}$. Since $\mathcal{A}_{n}$ is of index 2 in $\mathcal{S}_{n}$, we have that $\mathcal{S}_{n}^{[d]}=\mathcal{S}_{n}$.

By Theorem 3.11, we have the following result, which gives a summary of the discussion done so far in this subsection.

Theorem 4.1. Let $\pi$ be a (possibly infinite) supernatural number and let $\mathrm{H}_{\pi}$ be the corresponding pseudovariety of abelian groups. Then

(1) $\mathrm{K}_{\mathrm{H}_{\pi}}\left(\mathrm{C}_{n}\right)=\left\langle g^{d}\right\rangle$, with $d=\operatorname{gcd}(n, \pi)$. Furthermore, $\left|\mathrm{K}_{\mathrm{H}_{\pi}}\left(\mathrm{C}_{n}\right)\right|=n / d$.

(2) If $\pi$ is even, then $\mathrm{K}_{\mathrm{H}_{\pi}}\left(\mathrm{D}_{2 n}\right)=\mathrm{K}_{\mathrm{Ab}}\left(\mathrm{D}_{2 n}\right)=\mathrm{D}_{2 n}^{\prime}=\left\langle g^{2}\right\rangle$. If $\pi$ is odd, then $\mathrm{K}_{\mathrm{H}_{\pi}}\left(\mathrm{D}_{2 n}\right)=\mathrm{D}_{2 n}$.

(3) If $\pi$ is even, then $\mathrm{K}_{\mathrm{H}_{\pi}}\left(\mathcal{S}_{n}\right)=\mathrm{K}_{\mathrm{Ab}}\left(\mathcal{S}_{n}\right)=\mathcal{A}_{n}$. If $\pi$ is odd, then $\mathrm{K}_{\mathrm{H}_{\pi}}\left(\mathcal{S}_{n}\right)=\mathcal{S}_{n}$.

4.1.4. The case of an abelian group. Let $G$ be a finite abelian group. By Theorem 2.3, $G$ is isomorphic to a direct product $\mathrm{C}_{m_{1}} \times \mathrm{C}_{m_{2}} \times \cdots \times \mathrm{C}_{m_{t}}$, where the $\mathrm{C}_{m_{i}}$ are cyclic groups of order $m_{i}$ generated by, say, $g_{i}$ (with $1 \leq i \leq t$ ). A set of generators of $G$ is $\left\{\left(g_{1}, 0, \ldots, 0\right), \ldots,\left(0, \ldots, 0, g_{t}\right)\right\}$. Let $d=\operatorname{gcd}(\pi,|G|)$. Since $G$ is abelian and $G^{[d]}$ is generated by the $d$-powers of a set of generators, it follows easily that $G^{[d]}$ is $\mathrm{C}_{m_{1}}^{[d]} \times \mathrm{C}_{m_{2}}^{[d]} \times$ $\cdots \times \mathrm{C}_{m_{t}}^{[d]}$, which, by Lemma 3.1 , is $\mathrm{C}_{m_{1}}^{\left[d_{1}\right]} \times \mathrm{C}_{m_{2}}^{\left[d_{2}\right]} \times \cdots \times \mathrm{C}_{m_{t}}^{\left[d_{t}\right]}$, where $d_{i}=\operatorname{gcd}\left(m_{i}, \pi\right)$, with $1 \leq i \leq t$

Theorem 4.2. With the notation introduced, we have that $\mathrm{K}_{\mathrm{H}_{\pi}}(G)=\left\langle g_{1}^{d_{1}}\right\rangle \times \cdots \times\left\langle g_{t}^{d_{t}}\right\rangle$.

We may suppose that $\mathrm{C}_{m_{1}} \times \mathrm{C}_{m_{2}} \times \cdots \times \mathrm{C}_{m_{t}}$ is the canonical form for the group $G$ given by the fundamental theorem of finite abelian groups. Recall that this means that, for $i \in\{1, \ldots, t-1\}, m_{i+1}$ divides $m_{i}$, that is, there exist $q_{i}$ such that $m_{i}=m_{i+1} q_{i}$. Then there exist a divisor $q_{i}^{\prime}$ of $q_{i}$ such that $d_{i}=\operatorname{gcd}\left(m_{i}, \pi\right)=\operatorname{gcd}\left(m_{i+1} q_{i}, \pi\right)=\operatorname{gcd}\left(m_{i+1}, \pi\right) q_{i}^{\prime}=$ $d_{i+1} q_{i}^{\prime}$. It follows that

$$
\frac{m_{i}}{d_{i}}=\frac{m_{i+1} q_{i}}{d_{i+1} q_{i}^{\prime}}=\frac{m_{i+1}}{d_{i+1}} \cdot \frac{q_{i}}{q_{i}^{\prime}} .
$$

As a consequence, we have the following:

Remark 4.3. The computation of $\mathrm{K}_{\mathrm{H}_{\pi}}(G)$, as performed by Theorem 4.2, gives directly the canonical form for the group $\mathrm{K}_{\mathrm{H}_{\pi}}(G)$ given by the fundamental theorem of the finite abelian groups, provided that $G$ is also given through this form.

\subsection{Iterating the relative abelian kernel operator.}

4.2.1. A set of divisors of a supernatural number. Let $n$ be a positive integer and let $\pi$ be an infinite supernatural number, or a natural number greater than 1 . Let us consider the sequence $\left(d_{i}\right)_{i \geq 1}$, defined recursively as follows:

$$
\left\{\begin{array}{l}
d_{1}=\operatorname{gcd}(n, \pi) \\
d_{i+1}=\operatorname{gcd}\left(\frac{n}{d_{1} d_{2} \cdots d_{i}}, \pi\right), \text { for } i \geq 1 .
\end{array}\right.
$$


Clearly, there exists $j \in \mathbb{N}$ such that $d_{i}=1$, for all $i \geq j$. Let $i_{0}$ be the least such $j$ and denote

$$
\delta_{\pi, n}=\left\{d_{1}, \ldots, d_{i_{0}}\right\} .
$$

It is a decreasing chain of divisors of $\pi$. Note that $\prod_{d_{i} \in \delta_{\pi, n}} d_{i}$ divides $n$.

Example 4.4. (1) Let $n=2^{5} \cdot 3^{2} \cdot 5^{3} \cdot 11 \cdot 13$ and $\pi=2 \cdot 3^{+\infty} \cdot 5^{2} \cdot 7^{+\infty} \cdot 11^{3} \cdot 13^{+\infty}$.

(Notice that all the prime divisors of $n$ also divide $\pi$.) Then

$$
\begin{aligned}
& d_{1}=\operatorname{gcd}(n, \pi)=2 \cdot 3^{2} \cdot 5^{2} \cdot 11 \cdot 13 \\
& d_{2}=\operatorname{gcd}\left(2^{4} \cdot 5, \pi\right)=2 \cdot 5 \\
& d_{3}=\operatorname{gcd}\left(2^{3}, \pi\right)=2 \\
& d_{4}=\operatorname{gcd}\left(2^{2}, \pi\right)=2 \\
& d_{5}=\operatorname{gcd}(2, \pi)=2 \\
& d_{i}=\operatorname{gcd}(1, \pi)=1, \text { for } i \geq 6 .
\end{aligned}
$$

We have $\delta_{\pi, n}=\left\{2 \cdot 3^{2} \cdot 5^{2} \cdot 11 \cdot 13,2 \cdot 5,2,2,2,1\right\}$ and $n=\prod_{d_{i} \in \delta_{\pi, n}} d_{i}$.

(2) Let $n=3^{2} \cdot 5^{3} \cdot 7^{2} \cdot 11 \cdot 13$ and $\pi=2 \cdot 3^{+\infty} \cdot 5^{2} \cdot 11^{3} \cdot 13^{+\infty}$. Note that there are divisors of $n$ that do not divide $\pi$. In this case we have $\delta_{\pi, n}=\left\{3^{2} \cdot 5^{2} \cdot 11 \cdot 13,5,1\right\}$ and $n>\prod_{d_{i} \in \delta_{\pi, n}} d_{i}$.

This example motivates the following lemma.

Lemma 4.5. Let $\delta_{\pi, n}$ be as defined above. All the prime divisors of $n$ divide $\pi$ if and only if $n=\prod_{d_{i} \in \delta_{\pi, n}} d_{i}$.

Proof. If $n=\prod_{d_{i} \in \delta_{\pi, n}} d_{i}$, it is clear that all prime divisors of $n$ divide $\pi$, since any element of $\delta_{\pi, n}$ divides $\pi$.

Conversely, if $\prod_{d_{i} \in \delta_{\pi, n}} d_{i}<n$, then there exists a factor $q>1$ of $n$ such that $n=$ $\prod_{d_{i} \in \delta_{\pi, n}} d_{i} \cdot q$. But then $q$ is a divisor of $n$ that does not divide $\pi$.

4.2.2. The case of a finite cyclic group. We make use of the sequence (6) introduced in previous subsection. Note that $\mathrm{K}_{\mathrm{H}_{\pi}}\left(\mathrm{C}_{n}\right)=\left\langle g^{d_{1}}\right\rangle$. Iterating, we have $\mathrm{K}_{\mathrm{H}_{\pi}}^{s}\left(\mathrm{C}_{n}\right)=\left\langle g^{d_{1} d_{2} \cdots d_{s}}\right\rangle$. It follows that $\mathrm{C}_{n}$ is $\mathrm{H}_{\pi}$-solvable if and only if $n=\prod_{d_{i} \in \delta_{\pi, n}} d_{i}$. Using Lemma 4.5, we can conclude that in the case of a cyclic group the converse of Corollary 3.15 also holds:

Proposition 4.6. The cyclic group $\mathrm{C}_{n}$ is $\mathrm{H}_{\pi}$-solvable if and only if all the prime divisors of $n$ divide $\pi$.

Let $\mathrm{C}_{n}$ be a $\mathrm{H}_{\pi}$-solvable cyclic group of order $n=p_{1}^{t_{1}} p_{2}^{t_{2}} \cdots p_{s}^{t_{s}}$ where the $p_{i}$ are primes and the $t_{i}(1 \leq i \leq s)$ are positive integers. By the previous proposition $\pi$ may be written in the form $p_{1}^{\alpha_{1}} p_{2}^{\alpha_{2}} \cdots p_{s}^{\alpha_{s}} \cdot \pi_{1}$, where $\pi_{1}$ is a supernatural number and the $\alpha_{i}(0 \leq i \leq s)$ are positive integers or $+\infty$. Given integers $t$ and $\alpha$, we denote by $\left\lceil\frac{t}{\alpha}\right\rceil$ the least integer non smaller than $\frac{t}{\alpha}$ and use the convention $\left\lceil\frac{t}{+\infty}\right\rceil=1$. Recall that the notation $\ell_{\mathbf{H}}(G)$ is used for the $\mathrm{H}$-kernel chain length of the group $G$. We have proved the following:

Theorem 4.7. With the notation introduced, $\ell_{\mathbf{H}_{\pi}}\left(\mathrm{C}_{n}\right)=\max _{1 \leq i \leq r}\left(\left\lceil\frac{t_{i}}{\alpha_{i}}\right\rceil\right)$.

4.2.3. The case of a finite dihedral group. As a consequence of Corollary 3.14 or of The-

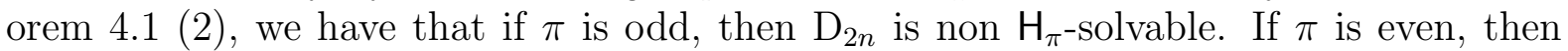
$\mathrm{K}_{\mathrm{H}_{\pi}}\left(\mathrm{D}_{2 n}\right.$ ) is cyclic (of order $n$ or $n / 2$, according to the parity of $n$ ), by Theorem 4.1 . Using Proposition 4.6, we have:

Proposition 4.8. The dihedral group $\mathrm{D}_{2 n}$ is $\mathrm{H}_{\pi}$-solvable if and only if all prime divisors of $2 n$ divide $\pi$. 
Since we can compute the $\mathrm{H}_{\pi}$-kernel chain length of a $\mathrm{H}_{\pi}$-solvable cyclic group, by Theorem 4.7, we can also compute the $\mathrm{H}_{\pi}$-kernel chain length of a solvable dihedral group.

Corollary 4.9. Let $\mathrm{D}_{2 n}$ be a $\mathrm{H}_{\pi}$-solvable dihedral group. The following holds:

$$
\ell_{\mathrm{H}_{\pi}}\left(\mathrm{D}_{2 n}\right)=\left\{\begin{array}{l}
1+\ell_{\mathrm{H}_{\pi}}\left(\mathrm{C}_{n}\right) \\
1+\ell_{\mathrm{H}_{\pi}}\left(\mathrm{C}_{n / 2}\right)
\end{array} \quad \text { if } n \text { is } n \text { is even } .\right.
$$

4.2.4. The case of a finite abelian group. In the remaining of this section, $G$ denotes a finite abelian group. We will assume that $G=\mathrm{C}_{m_{1}} \times \mathrm{C}_{m_{2}} \times \cdots \times \mathrm{C}_{m_{t}}$ is in the canonical form given by the fundamental theorem of finite abelian groups.

The simple observation that $G^{[m]}=\left\langle\left\{g^{m} \mid g \in A\right\}\right\rangle$, for a generating set $A$ of $G$, made at the beginning of this section, has important consequences. One of them is the following:

Corollary 4.10. Let $r_{1}, \ldots, r_{s}$ be positive integers. Then $\left(\cdots\left(G^{\left[r_{1}\right]}\right)^{\left[r_{2}\right]} \cdots\right)^{\left[r_{s}\right]}=G^{\left[r_{1} \cdots r_{s}\right]}$.

Using Remark 4.3, we get easily the following result, which reduces the computation of the $\mathrm{H}_{\pi}$-kernel chain length of a solvable abelian group to the computation of the $\mathrm{H}_{\pi}$-kernel chain length of a solvable cyclic group, which we can do by using Theorem 4.7.

Corollary 4.11. Let $\mathrm{C}_{m_{1}} \times \mathrm{C}_{m_{2}} \times \cdots \times \mathrm{C}_{m_{t}}$ be the canonical form for the finite abelian group $G$ given by the fundamental theorem of finite abelian groups and suppose that $G$ is $\mathrm{H}_{\pi}$-solvable. Then

$$
\ell_{\mathbf{H}_{\pi}}(G)=\ell_{\mathbf{H}_{\pi}}\left(\mathrm{C}_{m_{1}}\right) .
$$

4.2.5. The case of a finite symmetric group. By Theorem 4.1(3) or by Corollary 3.14, if $\pi$ is odd, then $\mathcal{S}_{n}$ is non $\mathrm{H}_{\pi}$-solvable.

If $\pi$ is even, then, by Theorem 4.1(3), we have that $\mathrm{K}_{\mathrm{H}_{\pi}}\left(\mathcal{S}_{n}\right)=\mathcal{A}_{n}$. As, for $n \geq 5$, the alternating group $\mathcal{A}_{n}$ is simple, then $\mathrm{K}_{\mathrm{Ab}}\left(\mathcal{A}_{n}\right)=\mathcal{A}_{n}$ and $\mathrm{K}_{\mathrm{Ab}}\left(\mathcal{A}_{n}\right) \subseteq \mathrm{K}_{\mathrm{H}_{\pi}}\left(\mathcal{A}_{n}\right)$, thus $\mathrm{K}_{\mathrm{H}_{\pi}}^{2}\left(\mathcal{S}_{n}\right)=\mathrm{K}_{\mathrm{H}_{\pi}}\left(\mathcal{A}_{n}\right)=\mathcal{A}_{n}$, showing that $\mathcal{S}_{n}$ is non $\mathrm{H}_{\pi}$-solvable.

Proposition 4.12. For $n \geq 5$, the symmetric group $\mathcal{S}_{n}$ is non $\mathrm{H}_{\pi}$-solvable.

It remains to check what happens for $n=2,3,4$.

We have $\mathcal{S}_{2} \cong C_{2}, \mathcal{S}_{3} \cong D_{6}$, thus the cases $n=2,3$ have already been studied. It remains to check how the $\mathrm{H}_{\pi}$-solvability of $\mathcal{S}_{4}$ depends on $\pi$. As $\mathrm{K}_{\mathrm{H}_{\pi}}^{2}\left(\mathcal{S}_{4}\right)=\mathrm{K}_{\mathrm{H}_{\pi}}\left(\mathcal{A}_{4}\right)=$ $\mathcal{A}_{4}^{[\operatorname{gcd}(12, \pi)]}$, we should start by computing $\mathcal{A}_{4}^{[d]}$, with $d \in\{2,4,6,12\}$.

The following GAP [12] session, which contains well known or easily verifiable facts concerning $\mathcal{A}_{4}$, is here for the convenience of the reader. It may turn the next two paragraphs more agreable to read.

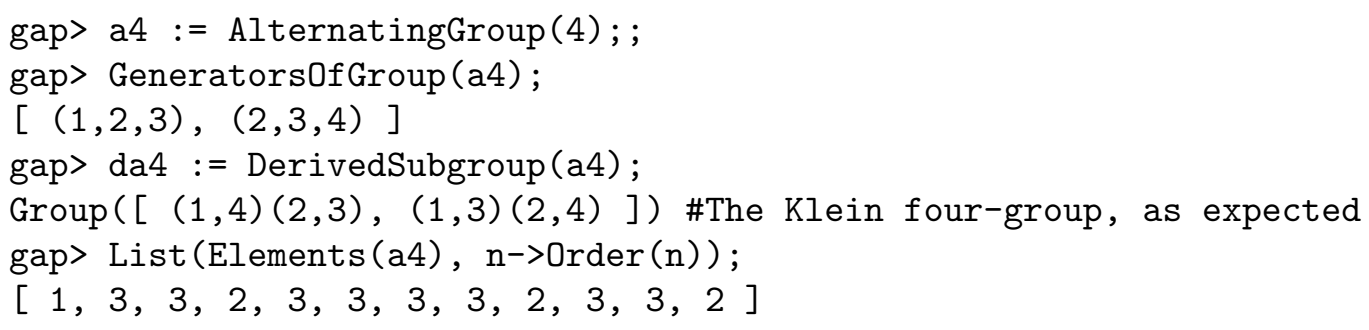

The alternating group $\mathcal{A}_{4}$ is generated by cycles of length 3 . If $\sigma$ is a cycle of length 3 and $k$ is an integer not divisible by 3 , then $\sigma^{k}$ is also a cycle of length 3 . Furthermore, any cycle of length 3 may be written as the square (and consequently as the fourth power) of a cycle of length 3 . As a consequence, we have that $\mathcal{A}_{4}^{[2]}=\mathcal{A}_{4}^{[4]}=\mathcal{A}_{4}$. 
The derived subgroup of $\mathcal{A}_{4}$ is $V=\mathrm{C}_{2} \times \mathrm{C}_{2}$, the Klein four-group, as is well known. The possible orders of the elements of $\mathcal{A}_{4}$ are 1,2 or 3 , which implies that $\mathcal{A}_{4}^{[6]}=\mathcal{A}_{4}^{[12]}=V$. As $V$ is abelian and its elements have orders not greater than 2 we have that $V^{[d]}=1$ if $d$ is even. Therefore, $\mathcal{S}_{4}$ is $\mathrm{H}_{\pi}$-solvable if and only if 6 divides $\pi$.

\section{ON THE $\mathrm{H}_{\pi}$-SOLVABILITY OF SOME INVERSE MONOIDS}

In this section we give upper bounds for the number of iterations of the relative abelian kernel operator needed to reach the idempotents of some monoids of injective partial transformations. It is inspired in part of the paper [5] by Fernandes and the second author, where the question has been treated for the same monoids and the (non relative) abelian kernel.

Let $n>1$ be a positive integer.

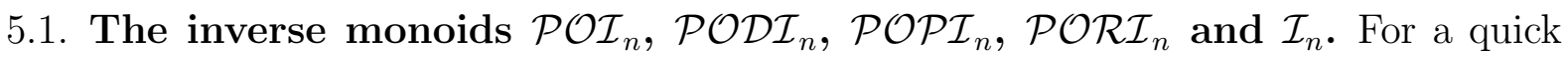
survey on these monoids, we suggest the paper [10] by Fernandes or any of the papers closely related to the present one: $[5,1]$. References for a deeper understanding of these monoids can be found there.

Let $M \in\left\{\mathcal{I}_{n}, \mathcal{P O} \mathcal{I}_{n}, \mathcal{P O D} \mathcal{I}_{n}, \mathcal{P O P} \mathcal{I}_{n}, \mathcal{P O} \mathcal{O} \mathcal{I}_{n}\right\}$. Then

$$
M / \mathscr{J}=\left\{J_{0} \leq \mathscr{J} J_{1} \leq \mathscr{J} \cdots \leq_{\mathscr{J}} J_{n}\right\},
$$

where, for each $0 \leq k \leq n, J_{k}$ is the $\mathscr{J}$-class consisting of the transformations $s$ such that $|\operatorname{Im}(s)|=k$. Concerning maximal subgroups, the monoid $\mathcal{P O} \mathcal{I}_{n}$ is aperiodic, while each $\mathscr{H}$-class of an element $s \in \mathcal{P O O D} \mathcal{I}_{n}$ has exactly two elements (an order-preserving one and another being order-reversing), unless the rank of $s$ is one or zero, in which case its $\mathscr{H}$-class is trivial. On the other hand, for $1 \leq k \leq n$, the $\mathscr{H}$-class of an element $s \in \mathcal{P O P} \mathcal{I}_{n}$ of rank $k$ has precisely $k$ elements, being a cyclic group of order $k$ if $s$ is a group-element. Finally, given $s \in \mathcal{P O} \mathcal{R} \mathcal{I}_{n}$, if $|\operatorname{Im}(s)|=k \geq 3$, then the $\mathscr{H}$-class of $s$ has $2 k$ elements and, if $s$ is a group-element, it is isomorphic to the dihedral group $D_{2 k}$ and if $|\operatorname{Im}(s)|=2$ then $\mathscr{H}$-class of $s$ has precisely two elements, otherwise it has just one element.

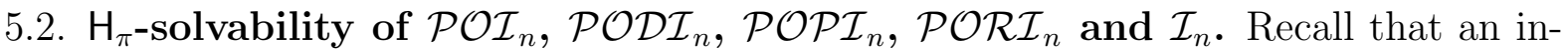
verse monoid $M$ is $\mathrm{H}$-solvable if and only if, for each idempotent $e \in M$, the maximal subgroup $H_{e}$ of $M$ containing $e$ is $\mathrm{H}$-solvable.

Proposition 5.1. Let $\pi$ be a (possibly infinite) supernatural number and let $\mathrm{H}_{\pi}$ be the corresponding pseudovariety of abelian groups. Then

(1) $\mathcal{P O \mathcal { I } _ { n }}$ is $\mathrm{H}_{\pi}$-solvable.

(2) $\mathcal{P O P} \mathcal{I}_{n}$ is $\mathrm{H}_{\pi}$-solvable if and only if all the prime numbers not greater than $n$ divide $\pi$.

(3) If $\pi$ is even, then $\mathcal{P O D} \mathcal{I}_{n}$ is $\mathrm{H}_{\pi}$-solvable.

If $\pi$ is odd, then $\mathcal{P O D} \mathcal{I}_{n}$ is non $\mathrm{H}_{\pi}$-solvable.

(4) If $\pi$ is even, then $\mathcal{P O R} \mathcal{I}_{n}$ is $\mathrm{H}_{\pi}$-solvable, if all the prime numbers not greater than $n$ divide $\pi$.

If $\pi$ is odd, then $\mathcal{P O R} \mathcal{I}_{n}$ is non $\mathrm{H}_{\pi}$-solvable.

(5) If $n \geq 5$, then $\mathcal{I}_{n}$ is non $\mathrm{H}_{\pi}$-solvable, independently of the parity of $\pi$.

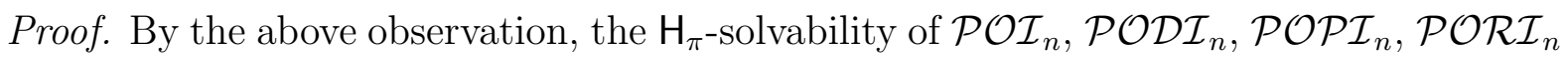
and $\mathcal{I}_{n}$ only depends on the $\mathrm{H}_{\pi}$-solvability of its maximal subgroups. These have been studied in Subsection 4.2. 
(1) The monoid $\mathcal{P O} \mathcal{I}_{n}$ is aperiodic, thus its subgroups are trivial. Therefore $\mathcal{P O} \mathcal{I}_{n}$ is $\mathrm{H}_{\pi}$-solvable.

(2) All the subgroups of the monoid $\mathcal{P O P} \mathcal{I}_{n}$ are cyclic. Their orders go from 1 to $n$. Using Proposition 4.6, we conclude that $\mathcal{P} \mathcal{O P} \mathcal{I}_{n}$ is $\mathrm{H}_{\pi}$-solvable if and only if all the prime numbers not greater than $n$ are divisors of $\pi$.

(3) The subgroups of $\mathcal{P} \mathcal{O D} \mathcal{I}_{n}$ have orders not greater than 2 , therefore are cyclic. Using Proposition 4.6 again, we conclude that $\mathcal{P O P} \mathcal{I}_{n}$ is $\mathrm{H}_{\pi}$-solvable if and only if $\pi$ is even.

(4) The maximal subgroups of $\mathcal{P O} \mathcal{R} \mathcal{I}_{n}$ are dihedral of order $2 k$, with $k \in\{1, \ldots, n\}$. By Proposition 4.8, if $\pi$ is odd, none of the $\mathrm{D}_{2 k}$ is $\mathrm{H}_{\pi}$-solvable. If $\pi$ is even, using Proposition 4.8 again, we have that the maximal subgroups $\mathrm{D}_{2 k}$ are $\mathrm{H}_{\pi \text {-solvable }}$ exactly when all the prime divisors of $k$ divide $\pi$. It follows that all the maximal subgroups of $\mathcal{P O} \mathcal{R} \mathcal{I}_{n}$ are $\mathrm{H}_{\pi}$-solvable if and only all the primes not greater than $n$ divide $\pi$.

(5) The maximal subgroups of $\mathcal{I}_{n}$ are symmetric of order $k$, with $k \in\{1, \ldots, n\}$, thus, by Proposition 4.12 , we conclude that, for $n \geq 5, \mathcal{I}_{n}$ is non $\mathrm{H}_{\pi}$-solvable.

As a consequence of Proposition 5.1 (2) we have that if $\mathrm{H}_{\pi} \neq \mathrm{Ab}$, the pseudovariety POPI contains non $\mathrm{H}_{\pi}$-solvable monoids.

5.3. On the length of relative abelian kernel chains of a relatively solvable monoid. We follow the notation introduced in [5]. For a non-trivial $\mathscr{J}$-class $J$ of $M$ denote by $\ell(J)$ the maximum length of a $\leq \mathscr{J}$-chain of non-trivial $\mathscr{J}$-classes of $M$ having $J$ as the $\leq \mathscr{J}$-maximum. Denote by $\Omega(J)$ the subset of $M / \mathscr{J}$ of all non-trivial $\mathscr{J}$ classes $I$ such that $\ell(I)=\ell(J)$ and by $\lambda(J)$ the maximum of the $\mathrm{H}_{\pi}$-kernel lengths of the maximal subgroups of $M$ contained in the members of $\Omega(J)$. Let

$$
\tilde{\lambda}(J)= \begin{cases}1 & \text { if all the groups in } J \text { are trivial; } \\ \lambda(J) & \text { otherwise. }\end{cases}
$$

The following result, which will allow us to give an upper bound for the length of the $\mathrm{H}_{\pi^{-}}$ kernel chain of some inverse monoids, is a generalization of [5, Proposition 2.1], proved by Fernandes and the second author. Its proof, consisting mostly of straightforward generalizations of results from [5], can be checked in [2].

Proposition 5.2. Let $M$ be a solvable inverse monoid and let $\left\{J_{1} \leq \mathscr{J} J_{2} \leq \mathscr{J} \cdots \leq \mathscr{J}\right.$ $\left.J_{k}\right\}$ be $a \leq \mathscr{J}$-chain of maximum size of non-trivial $\mathscr{J}$-classes of $M$. Then $\ell_{\mathbf{H}_{\pi}}(M) \leq$ $\sum_{i=1}^{k} \tilde{\lambda}(J)$.

5.3.1. The case of the monoid $\mathcal{P O} \mathcal{I}_{n}$. As all the subgroups of $\mathcal{P O} \mathcal{I}_{n}$ are trivial, we have $\lambda\left(J_{k}\right)=0$. Then, by Proposition $5.2, \ell_{\mathbf{H}_{\pi}}\left(\mathcal{P} \mathcal{O I}_{n}\right) \leq n-1$. Note that $n-1$ is the number of non-trivial $\mathscr{J}$-classes of $\mathcal{P} \mathcal{O I}_{n}$.

5.3.2. The case of the monoid $\mathcal{P O D} \mathcal{I}_{n}$. By Proposition 5.1, $\mathcal{P O D} \mathcal{I}_{n}$ is $\mathrm{H}_{\pi}$-solvable exactly when the supernatural number $\pi$ is even. As $\mathcal{P O D} \mathcal{I}_{n}$ has $n$ non-trivial $\mathscr{J}$-classes and the maximal subgroups contained in these $\mathscr{J}$-classes are of order 2 , we have that $\lambda\left(J_{i}\right)=1$, for any $1 \leq i \leq n$. As a consequence we have that $\ell_{\mathrm{H}_{\pi}}\left(\mathcal{P} \mathcal{O D} \mathcal{I}_{n}\right) \leq n$. Another way to obtain the same upper bound is to use the fact (see [1]) that $\mathrm{K}_{\mathrm{H}_{\pi}}\left(\mathcal{P O D} \mathcal{I}_{n}\right) \subseteq$ $\mathcal{P O \mathcal { I } _ { n }}$ and the above result concerning the monoid $\mathcal{P O} \mathcal{I}_{n}$. 
5.3.3. The case of the monoid $\mathcal{P O P \mathcal { I }}{ }_{n}$. Recall that, by Proposition 5.1, $\mathcal{P O P} \mathcal{I}_{n}$ is $\mathrm{H}_{\pi^{-}}$ solvable if and only if $\pi$ is a supernatural number that is divisible by all the prime numbers not greater than $n$. As usual, denote by $J_{k}$ the $\mathscr{J}$ class consisting of the transformations of rank $k$ of $\mathcal{P O P} \mathcal{I}_{n}$.

Since the subgroups of $J_{k}$ are cyclic of order $k$, Theorem 4.7 leads to the following:

Proposition 5.3. Let $p_{1}, \ldots, p_{r}$ be the primes not greater then $n$ and let $\pi=p_{1}^{\alpha_{1}} p_{2}^{\alpha_{2}} \cdots p_{r}^{\alpha_{r}} \pi_{1}$ be a supernatural number, with the $\alpha_{i}$ positive integers, for $1 \leq i \leq r$. For $1 \leq k \leq n$, we have $k=p_{1}^{t_{k, 1}} p_{2}^{t_{k, 2}} \cdots p_{r}^{t_{k, r}}$, for some non negative integers $t_{k, i}$, with $1 \leq i \leq r$. Then

a) $\lambda\left(J_{k}\right)=\max _{1 \leq i \leq r}\left(\left\lceil\frac{t_{k, i}}{\alpha_{i}}\right\rceil\right)$.

b) $\ell_{\mathbf{H}_{\pi}}\left(\mathcal{P} \mathcal{O P} \mathcal{I}_{n}\right) \leq \sum_{k=1}^{n} \tilde{\lambda}\left(J_{k}\right)$.

5.3.4. The case of the monoid $\mathcal{P O} \mathcal{R} \mathcal{I}_{n}$. Recall that, by Proposition 5.1, $\mathcal{P O} \mathcal{R} \mathcal{I}_{n}$ is $\mathrm{H}_{\pi^{-}}$ solvable if and only if $\pi$ is an even supernatural number that is divisible by all the prime numbers not greater than $n$. As usual, denote by $J_{k}$ the $\mathscr{J}$ class consisting of the transformations of rank $k$ of $\mathcal{P O R} \mathcal{I}_{n}$.

Since the subgroups of $J_{k}$ are dihedral of order $k$, Corollary 4.9 leads to the following:

Proposition 5.4. Let $p_{1}, \ldots, p_{r}$ be the primes not greater than $n$ and let $\pi=p_{1}^{\alpha_{1}} p_{2}^{\alpha_{2}} \cdots p_{r}^{\alpha_{r}} \pi_{1}$ be a supernatural number, with the $\alpha_{i}$ positive integers, for $1 \leq i \leq r$. For $1 \leq k \leq n$, we have $k=p_{1}^{t_{k, 1}} p_{2}^{t_{k, 2}} \cdots p_{r}^{t_{k, r}}$, for some non negative integers $t_{k, i}$, with $1 \leq i \leq r$. Then
a) $\lambda\left(J_{k}\right)=\left\{\begin{array}{l}1+\ell_{\mathbf{H}_{\pi}}\left(\mathrm{C}_{k}\right) \\ 1+\ell_{\mathrm{H}_{\pi}}\left(\mathrm{C}_{k / 2}\right)\end{array} \quad\right.$ if $k$ is $k$ is oven.
b) $\ell_{\mathrm{H}_{\pi}}\left(\mathcal{P O} \mathcal{R} \mathcal{I}_{n}\right) \leq \sum_{k=1}^{n} \tilde{\lambda}\left(J_{k}\right)$.

\section{ACKNOWLEDGEMENTS}

This work was partly supported by Fundação para a Ciência e a Tecnologia (FCT) through the Centro de Matemática da Universidade do Porto (CMUP) and by the project PTDC/MAT/65481/2006, which is partly funded by the European Community Fund FEDER. The second author gratefully acknowledges also the ESF programme "Automata: from Mathematics to Applications (AutoMathA)" within whose framework the work leading to this paper has been carried out.

\section{REFERENCES}

[1] E. Cordeiro, M. Delgado, and V. H. Fernandes. Relative abelian kernels of some classes of transformation monoids. Bull. Austral. Math. Soc., 73(3):375-404, 2006.

[2] Edite Cordeiro. Núcleo e solubilidade de certos monóides inversos (relativamente a pseudovariedades de grupos abelianos). PhD thesis, Universidade do Porto, 2009. In Portuguese.

[3] Edite Cordeiro and Manuel Delgado. Computing relative abelian kernels of finite monoids. J. Algebra, 303(2):642-654, 2006.

[4] Manuel Delgado. Abelian pointlikes of a monoid. Semigroup Forum, 56(3):339-361, 1998.

[5] Manuel Delgado and Vítor H. Fernandes. Abelian kernels, solvable monoids and the abelian kernel length of a finite monoid. In V. Fernandes G. Gomes I. Araújo, M. Branco, editor, Semigroups and languages, pages 68-85. World Sci. Publ., River Edge, NJ, 2004.

[6] Manuel Delgado and Vítor H. Fernandes. Solvable monoids with commuting idempotents. Internat. J. Algebra Comput., 15(3):547-570, 2005.

[7] Manuel Delgado, Vítor H. Fernandes, Stuart Margolis, and Benjamin Steinberg. On semigroups whose idempotent-generated subsemigroup is aperiodic. Internat. J. Algebra Comput., 14(5-6):655665,2004 . International Conference on Semigroups and Groups in honor of the 65th birthday of Prof. John Rhodes. 
[8] Manuel Delgado and Benjamin Steinberg. On iterated Mal'cev products with a pseudovariety of groups. Technical Report CMUP 2008-32, CMUP, 2008.

[9] David S. Dummit and Richard M. Foote. Abstract algebra. John Wiley \& Sons Inc., Hoboken, NJ, third edition, 2004.

[10] Vítor H. Fernandes. Presentations for some monoids of partial transformations on a finite chain: a survey. In Semigroups, algorithms, automata and languages (Coimbra, 2001), pages 363-378. World Sci. Publ., River Edge, NJ, 2002.

[11] Joseph A. Gallian. Contemporary Abstract Algebra. Houghton-Mifflin, fifth edition, 2002.

[12] The GAP Group. GAP - Groups, Algorithms, and Programming, Version 4.4.11, 2008.

[13] Dinakar Ramakrishnan and Robert J. Valenza. Fourier analysis on number fields, volume 186 of Graduate Texts in Mathematics. Springer-Verlag, New York, 1999.

[14] Benjamin Steinberg. Monoid kernels and profinite topologies on the free abelian group. Bull. Austral. Math. Soc., 60(3):391-402, 1999.

[15] Howard Straubing. Families of recognizable sets corresponding to certain varieties of finite monoids. J. Pure Appl. Algebra, 15(3):305-318, 1979.

E-mail address, E. Cordeiro: emc@ipb.pt

Instituto Politécnico de Bragança, Escola Superior de Tecnologia e Gestão, Campus de Santa Apolónia, 5301-857 Bragança, Portugal

E-mail address, M. Delgado: mdelgado@fc.up.pt

Departamento de Matemática Pura e CMUP, Faculdade de Ciências, Universidade do Porto, Rua do Campo Alegre 687, 4169-007 Porto, Portugal 